\title{
Factores Tecnológicos que Influyen en la Obtención de una Beca
}

\author{
Martha Jiménez-García y María E. Tavera-Cortes \\ Instituto Politécnico Nacional - Unidad Profesional Interdisciplinaria de Ingeniería y Ciencias Sociales y \\ Administrativas. Av. Té 950 Granjas México, Iztacalco, 08400 D. F, México. \\ (e-mail: majimenez@ipn.mx; mtavera@ipn.mx)
}

Recibido May. 24, 2017; Aceptado Jul. 20, 2017; Versión final Ago. 18, 2017, Publicado Feb. 2018

\begin{abstract}
Resumen
El objetivo de este es analizar el impacto de factores académicos y tecnológicos que influyen en la obtención de una beca en estudiantes de nivel superior. El Banco Mundial y el Plan Nacional de Desarrollo en México 2013-2018 indican que se deben de fomentar las habilidades de las Tecnologías de Información y Comunicación en la educación para generar un mayor ingreso. Se realizó un modelo de regresión logística con datos de 1182 alumnos, usando las siguientes variables: endógena (beca) y como variables exógenas: (semestre y herramientas informáticas). Se encontró que el uso del software Haiku Deck y la participación en las redes sociales aumentan la probabilidad de obtener una beca. Se concluye que en las universidades se debe usar software con material multimedia y se capacite a los docentes para fomentar un mayor uso de las redes sociales en la educación.
\end{abstract}

Palabras clave: becas de estudio; redes sociales; educación superior; posgrado

\section{Technological Factors that Influence the Obtaining a Scholarship}

\begin{abstract}
The objective of this work is to analyze the impact of academic and technological factors that influence the obtaining of a scholarship for students of higher education. The World Bank and the National Development Plan for Mexico 2013-2018 indicate that Information and Communication Technology skills in education should be encouraged to generate higher income. A logistic regression model with data of 1182 students was carried out. The variables are: endogenous (scholarship) and exogenous variables: (semester and computer tools). The results found indicate that the use of Haiku Deck software and participation in social networks increase the probability of obtaining a scholarship. It is concluded that that use of multimedia software in the universities must be promoted and teachers should be trained to encourage greater use of social networks in education.
\end{abstract}

Keywords: fellowships; social networks; higher education; graduate studies 


\section{INTRODUCCIÓN}

El Banco Mundial ha mencionado que los programas nacionales de tecnología en los países son importantes, debido a que fomentan el uso de las Tecnologías de Información y Comunicación (TIC) en todas las áreas, esto último propicia que se tengan mayores habilidades de TIC, lo cual indica que al tener estas habilidades se incrementa la productividad laboral y a su vez la competitividad en las organizaciones (Banco Mundial, 2012). Asimismo, el Índice Mundial de Innovación mencionó la importancia de la innovación como fuente de desarrollo económico (INSEAD, 2012); dicho índice incluye como recursos para la innovación a la infraestructura de TIC consistente en hardware y software, y como resultados de innovación a la producción creativa; es así como las TIC a nivel mundial se han convertido en apoyos esenciales por ser un motor de crecimiento económico. Sin embargo, México se encuentra en un nivel bajo de innovación de acuerdo con el índice mencionado, lo cual podría ser un freno en el crecimiento económico por la falta de innovación; por lo cual es necesario impulsar el uso de las TIC en México y surge la necesidad de esta investigación; es decir se requiere conocer qué tipo de TIC son necesarias en la formación del capital humano, para que a mediano plazo sea utilizado en las organizaciones.

Por otro lado, en México el Plan Nacional de Desarrollo 2013-2018 menciona la importancia del capital humano calificado o especializado como una fuente de innovación y de crecimiento económico; por lo que es importante robustecer la educación con las habilidades necesarias a nivel global; pues actualmente se tiene una gran cantidad de información, pero no se tienen las herramientas informáticas o las habilidades necesarias para tener un manejo efectivo de la información; lo cual da un amplio soporte al uso de las TIC. Asimismo, de acuerdo con el Plan Nacional de Desarrollo de México una de las estrategias que se seguirán para aumentar la calidad educativa es una mayor inversión en ciencia y tecnología, básicamente se busca incluir nuevas tecnologías en los procesos de enseñanza y de aprendizaje con equipos de cómputo modernos (Gobierno de la República, 2013); por lo cual es importante encontrar las TIC adecuadas en cuanto a software que ayude a la educación a fortalecer las habilidades informáticas.

Independientemente de las habilidades informáticas, también indica el Plan Nacional de Desarrollo en México 20132018 que se fomente un aumento en la asignación del programa de becas de educación media superior y superior (Gobierno de la República, 2013); por lo que esta investigación también contribuye en encontrar aspectos que ayuden a obtener una beca de nivel superior. En México existen diferentes becas para el nivel superior, como la beca Telmex, becas institucionales, becas de manutención, becas de investigación, entre otras. Las becas son un estímulo económico para los alumnos para tener un mejor aprovechamiento académico, esto conduce hacia una educación de calidad; lo cual también conduce a tener personal más competitivo.

La iniciativa del Banco Mundial sobre fomentar el uso de las TIC y de incrementar el Índice Mundial de Innovación en México, así como lo planteado en el Plan Nacional de Desarrollo de México, sobre la inclusión de las TIC en la educación y un aumento en las becas, son aspectos que promueven la elaboración de este trabajo para encontrar los factores tecnológicos de TIC que den respuesta a las preguntas de investigación ¿Existe relación entre el uso de las TIC y el rendimiento académico para obtener una beca? ¿El uso de las TIC basadas en las redes sociales favorece la educación desde el punto de vista rendimiento académico? ¿Qué tipo de software favorece a los estudiantes para obtener una beca e incrementar su ingreso económico?, por lo que esta investigación se realizó en una universidad con enfoque multidisciplinario, lo cual hace que los resultados se puedan interpretar para cualquier universidad mexicana.

En esta investigación se presenta el resultado de un experimento mediante el uso de una encuesta sobre uso de las TIC en aspectos académicos que influyen en la obtención de una beca, lo cual lleva de forma directa el rendimiento académico, pues se requiere de cierto promedio para alcanzar una beca. La investigación es exploratoria y transversal. El objetivo de este trabajo es analizar el impacto de factores académicos y tecnológicos que influyen en la obtención de una beca estudiantil en alumnos de nivel superior. La hipótesis de este trabajo considera que la herramienta informática de creación de material multimedia denominada Haiku Deck y la participación en las redes sociales influye de forma positiva en la obtención de una beca estudiantil en alumnos de nivel superior.

Es importante el uso de las tecnologías informáticas en el ámbito académico, pues con el uso y el apoyo de las TIC se generan mayores habilidades informáticas que facilitan el aprendizaje. Asimismo, los profesores que tienen un amplio conocimiento y que usan las TIC, normalmente presentan un impacto positivo en la enseñanza basada en TIC (Fong y Holland, 2011; Peeraer y Van Petegem, 2011). Además, el proceso de aprendizaje se favorece con el uso de las TIC, que contienen material multimedia, pues la inclusión de los videos y los juegos facilita la comprensión de los contenidos de aprendizaje que son complejos (Alfaro et al., 2016; Martínez et al., 2015); lo cual hace que el alumno adquiera conocimiento de forma más agradable al percibir sonidos y apreciar imágenes. Sin embargo, hay que realizar una buena selección del material 
tecnológico que se utiliza, el cual debe corresponder al entorno de tipo social, económico y educativo en el que se encuentren los alumnos y los profesores, ya que este entorno condiciona a los participantes (Rodríguez, 2015).

Asimismo, la computadora es el medio más utilizado por la sociedad, pues permite utilizar diferentes aplicaciones informáticas para ser más productivos (Cabero-Almenara y Gutiérrez-Castillo, 2015), aunque actualmente la tendencia son los dispositivos móviles con la plataforma Android, por lo cual hay que analizar ambas tecnológicas las fijas y la móvil. Song et al., 2016 argumentan que el material con recursos educativos de tipo multimedia ayuda a los estudiantes a desarrollar una comprensión más completa de los contenidos de aprendizaje complejos, lo cual se ve reflejado en su rendimiento académico. Con la información de los autores mencionados se puede apreciar la importancia de las TIC en la educación, específicamente en la creación de material multimedia para facilitar los entornos académicos. Las TIC han penetrado en las actividades docentes de forma muy lenta, aunque en las escuelas privadas han penetrado de forma más exitosa que en las públicas; además los profesores de la instituciones privadas mencionan que el aprendizaje con el apoyo de las TIC es más efectivo que el enfoque tradicional; por lo que un mayor uso de los medios digitales (WhatsApp, foros, videoconferencias) también les ayuda a los profesores a tener una mayor comunicación en las actividades escolares con sus alumnos (Tapasco y Giraldo, 2017).

Las redes sociales actualmente están siendo utilizadas en todos los ámbitos y en todos los entornos económicos; los estudiantes de bajos ingresos se ven favorecidos al usar las redes sociales, pues obtienen información que no está disponible en contextos normales para ellos (Brown et al., 2016), lo cual propicia que se usen las redes sociales para compartir información a la cual no tienen acceso. Asimismo, en Arabia Saudita las redes sociales entre estudiantes universitarios presentan un impacto positivo en el aprendizaje con el uso del servicio de comunicación o chat en línea y el uso compartido de archivos; además las principales herramientas que utilizan los estudiantes en orden de preferencia son WhatsApp, YouTube y Facebook (Eid, y Al-Jabri, 2016). Lo cual indica que el uso de las redes sociales es importante para los alumnos para tener una mayor comunicación e intercambio de información, pues son usadas en varios países como Arabia Saudita siendo un país con Monarquía absoluta, pero con índice de desarrollo humano muy alto, lo cual indica que las redes sociales se emplean en cualquier ámbito sin importar la cultura de un país.

El uso del Facebook en el ámbito académico es básicamente para intercambiar información (Čičević et al., 2016). Asimismo, para un estudiante es más importante estar conectado con otros compañeros, que estar navegando por la web; pues los estudiantes presentan siempre una gran disponibilidad a los mensajes entrantes; y presentan un fuerte descontento cuando tienen pérdidas temporales de conexión a Internet (Vorderer et al., 2016). Igualmente, los estudiantes argumentan tener una actitud positiva con el uso Facebook cuando lo utilizan como complemento en las actividades del salón de clases, lo cual resulta ser eficaz para desarrollar un pensamiento crítico en la formación universitaria (Castro y González-Palta, 2016). Por lo cual se le debe de dar importancia al uso del Facebook para que el estudiante tenga un sentido de pertenencia al grupo académico y se mantenga en comunicación con sus pares académicos. Los estudiantes están de acuerdo con el uso de los blogs e indican que contribuye positivamente con el aprendizaje, pues expanden su aprendizaje más allá de las horas de clase y pueden interactuar con sus compañeros a cualquier hora, en cualquier lugar y donde ellos quieran hacerlo; esto también fomenta la cercanía emocional de los estudiantes con sus pares (Lee y Bonk, 2016).

En la educación superior se utiliza con mayor frecuencia una integración de tecnologías de la comunicación (TIC), por lo que los estudiantes perciben una mayor efectividad en el aprendizaje con el uso de dichas tecnologías utilizadas por sus profesores (Venkatesh et al., 2016), además el estudiante presenta un alto rendimiento académico con el uso innovador de las TIC (Durán, et al. 2017). Por lo cual es importante incluir las TIC en la educación como el Facebook, WhatsApp, YouTube, blogs, entre otros. El diseño de contenidos de las redes sociales para la educación debe ser atractivo, único, de fácil uso y debe incluir contenido para comprometer al estudiante en el aprendizaje (Mahapatra, 2016). Las redes sociales son una fuente de información que se utilizan diariamente y desempeñan un papel importante al proporcionar un mayor contenido de información en la educación, para las instituciones, los profesores y los estudiantes (Hamid et al., 2016).

Las redes sociales tienen un efecto positivo en el aprendizaje del estudiante, pues facilita la comunicación, además de motivar a los estudiantes para adquirir un nuevo conocimiento; asimismo, es conveniente utilizar las redes sociales, para dejar tareas a los alumnos que les fomenten el interés por tener información actualizada (Kitsantas et al., 2016). Con los autores previamente mencionados se ilustra de forma general la importancia de la participación en las redes sociales en el ámbito educativo, pues se obtiene información que no está disponible en contextos normales, además de que los universitarios les gusta usar el chat, discutir en tiempo real en cualquier lugar y situación, además prefieren las aplicaciones como WhatsApp, YouTube y Facebook, las cuales están disponibles en cualquier teléfono inteligente y esto los conduce a tener mayor productividad académica al tener una mayor comunicación. 


\section{METODOLOGIA}

La investigación fue de tipo exploratoria, realizada en la Ciudad de México, en una escuela de nivel superior del Instituto Politécnico Nacional. Específicamente en la Unidad Profesional Interdisciplinaria de Ingeniería y Ciencias Sociales y Administrativas (UPIICSA). Como instrumento de evaluación se aplicó una encuesta estructurada por 52 preguntas distribuidas por secciones. Cada una de las preguntas correspondía a una determinada herramienta informática. Para efectos de este documento se tomaron únicamente las variables que tenían correlación positiva con la existencia de una beca estudiantil; las becas de los alumnos son apoyos económicos que otorga el gobierno por las siguientes razones: 1) presentar alto rendimiento académico y 2) realizar actividades de investigación además de presentar alto rendimiento académico, asimismo existen becas que otorgan fundaciones de empresas privadas como la beca Telmex para mejorar el aprendizaje y de igual forma el requisito es tener una excelencia académica, además de tener una actividad extracurricular y un estudio socioeconómico; para todos los casos si el alumno no presenta un alto promedio académico, no puede tener derecho a la beca o apoyo económico. La encuesta se aplicó de forma aleatoria a los alumnos de licenciatura de las cinco diferentes carreras que oferta dicha universidad. La finalidad de este estudio fue investigar las herramientas informáticas que influyen de forma positiva en la existencia de una beca estudiantil.

La encuesta se aplicó a una muestra aleatoria de 1182 alumnos de una población aproximada de 13,000 durante el ciclo escolar 2015-2016. Asimismo, la encuesta obtuvo un alfa de Cronbach de 0.810, esto significa que existe consistencia interna a través de todos los elementos, por lo tanto, están correlacionados entre sí. Se pidió a los encuestados que señalaran que tan frecuentemente utilizaban las herramientas para buscar información en internet, las cuales fueron las indicadas por Viñas (2016). Se utilizó una escala que comprendió los números $0,2,3,5$ y 7 , los cuales indican la frecuencia de uso en días a la semana, para todas las variables exógenas. Las variables que tuvieron correlación positiva con la beca estudiantil se muestran en la Fig. 1, y se describen en la Tabla 1.

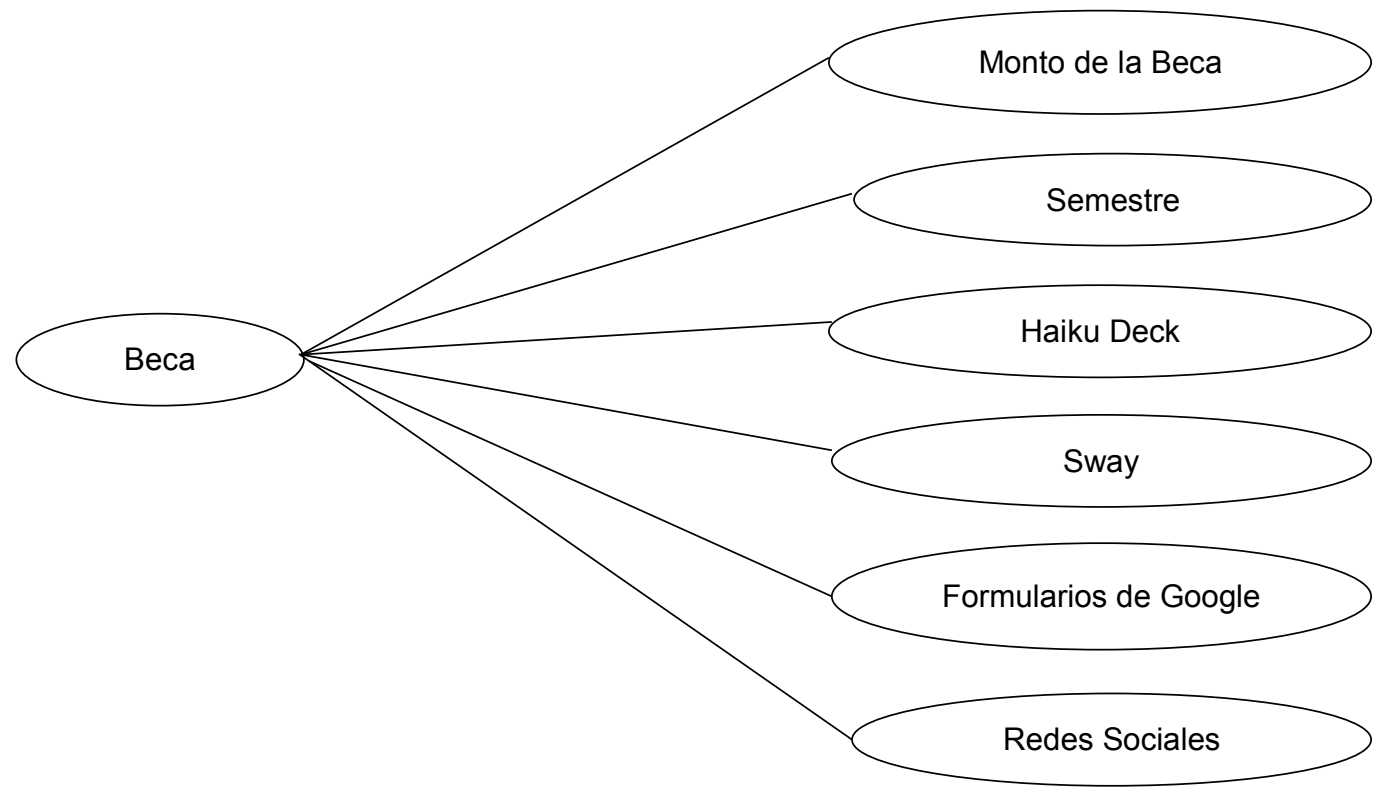

Fig. 1: Variables que influyen en la obtención de una beca estudiantil

Se utilizó un modelo de regresión Logit, el cual presenta solo dos alternativas posibles mutuamente excluyentes (éxito o fracaso -beca =éxito, no beca=fracaso), por lo que la variable endógena del modelo es binaria $(0,1)$. El modelo Logit se basa en una función de distribución logística para los errores aleatorios, y su estimación se hace a través del método de Máxima Verosimilitud. El análisis realizado se basa en el uso de un estadístico denominado valor-p, el cual corresponde a la probabilidad de aceptar la hipótesis nula, comparada con el nivel de significancia $\alpha$ (se utilizó $\alpha=0,05$ ). Asimismo, se utilizó una matriz de correlación de Pearson para establecer una primera aproximación sobre la determinación de las variables independientes, para conocer las variables que influyen en la existencia de una beca estudiantil. Se propone un modelo de regresión Logit, que contiene como variable endógena la existencia de una beca estudiantil y como variables exógenas: 1) semestre, las herramientas de creación de material multimedia; 2) Haiku Deck; 3) Sway, la herramienta para crear encuestas; 4) formularios de Google; y 5) participación en redes sociales.

En la ecuación 1, se muestra la probabilidad de que $Y=1$, y se denota por "p"; asimismo se presenta la relación con las variables dependientes. 


$$
p=\frac{1}{1+\exp \left(-\alpha-\beta_{1} X_{1}-\beta_{2} X_{2}-\beta_{3} X_{3}-\beta_{4} X_{4}-\beta_{5} X_{5}-\beta_{6} X_{6}\right)}
$$

En la ec. (1), la expresión "exp" denota la función exponencial y $\alpha, \beta_{1}, \beta_{2}, \beta_{3}, \beta_{4}, \beta_{5}$ y $\beta_{6}$, son los parámetros del modelo. Al resolver la función exponencial, $p$ tomará solo valores entre 0 y 1 . Si $\beta$ es positiva (mayor que 0 ) entonces la función es creciente y decreciente en el caso contrario.

Tabla 1: Descripción de variables que influyen para obtener una beca estudiantil

\begin{tabular}{ll}
\hline \multicolumn{1}{c}{ Variable } & \multicolumn{1}{c}{ Descripción } \\
\hline Beca $(\mathrm{Y})$ & $\begin{array}{l}\text { Remuneración económica otorgado por alguna } \\
\text { institución, en este caso la variable es } \\
\text { dicotómica, puede tomar el valor "0" si la beca } \\
\text { no ocurre y "1" si la beca ocurre }\end{array}$ \\
\hline Monto de la Beca & $\begin{array}{l}\text { Monto económico en pesos mexicanos } \\
\text { otorgada a un estudiante que reúne ciertos } \\
\text { requerimientos }\end{array}$ \\
\hline
\end{tabular}

Tipo y relación

Semestre $\left(\mathrm{X}_{2}\right)$

Semestre en el que está inscrito el alumno
Dependiente: Variable principal

Independiente: Se relaciona directamente con la variable dependiente de forma muy directa, pues es el incentivo más fuerte para un alumno el monto económico.

Independiente: Se relaciona con la variable beca, pues los estudiantes buscan todos los medios de obtener una beca conforme avanzan de semestre, al percibir que sus compañeros tienen una beca.

Haiku Deck una herramienta informática para crear
material multimedia. Herramienta web que
transforma la manera de presentar un contenido
para una retención más efectiva. La
presentación se apoya en imágenes para
amplificar el impacto emocional y memorable y
con el uso de pocas palabras para un mensaje
más claro y un formato limpio, claro y muy fácil
de configurar Viñas (2016).

Independientes: Estas son variables consideradas como tecnología, y se utilizan por considerar que el uso de la tecnología aumenta la productividad, en este caso es la productividad estudiantil, para buscar un mayor ingreso económico, lo cual se puede obtener a través de una beca.

\begin{tabular}{|c|c|}
\hline Sway $\left(\mathrm{X}_{4}\right)$ & $\begin{array}{l}\text { Es una herramienta informática para crear } \\
\text { material multimedia. Una aplicación web } \\
\text { innovadora de Microsoft que permite agrupar } \\
\text { contenidos interactivos (vídeos, imágenes, } \\
\text { entre otros) que encuentras en la web, creando } \\
\text { fácilmente una presentación multimedia para } \\
\text { capturar la atención de la clase. El diseño y } \\
\text { formato de la presentación funciona con un } \\
\text { simple arrastrar y soltar con sugerencias y } \\
\text { ajustes de estilo desde la misma aplicación } \\
\text { Viñas (2016). }\end{array}$ \\
\hline $\begin{array}{l}\text { Formularios de } \\
\text { Google }\left(X_{5}\right)\end{array}$ & $\begin{array}{l}\text { Crear encuestas y tests en tiempo real. Los } \\
\text { formularios de Google permiten planificar } \\
\text { eventos, enviar una encuesta, realizar } \\
\text { preguntas a los estudiantes o recopilar otros } \\
\text { tipos de información de forma fácil y eficiente. } \\
\text { Los formularios de Google pueden conectarse } \\
\text { a las hojas de cálculo de Google y las } \\
\text { respuestas se enviarán automáticamente a ella } \\
\text { para ver los resultados Viñas (2016). }\end{array}$ \\
\hline Redes Sociales $\left(X_{6}\right)$ & $\begin{array}{l}\text { Las redes sociales son grupos de personas } \\
\text { interconectadas de forma virtual que } \\
\text { interactúan entre si para intercambiar } \\
\text { información; las redes consideradas en esta } \\
\text { investigación son WhatsApp, YouTube, } \\
\text { Facebook, Google+ y Linkedin }\end{array}$ \\
\hline
\end{tabular}




\section{RESULTADOS}

En la Fig. 2 se presenta un diagrama de dispersión entre la variable TIC compuesta por las herramientas de software Haiku Deck, Sway, Formularios de Google y Redes Sociales, así como la variable Promedio (Rendimiento académico) la cual está directamente relacionada con la beca, pues para obtener una beca es necesario tener un promedio alto. Asimismo, se percibe una correlación positiva muy baja, por lo que se decide elaborar un modelo de regresión, para investigar cómo se comportan en el modelo de regresión logística.

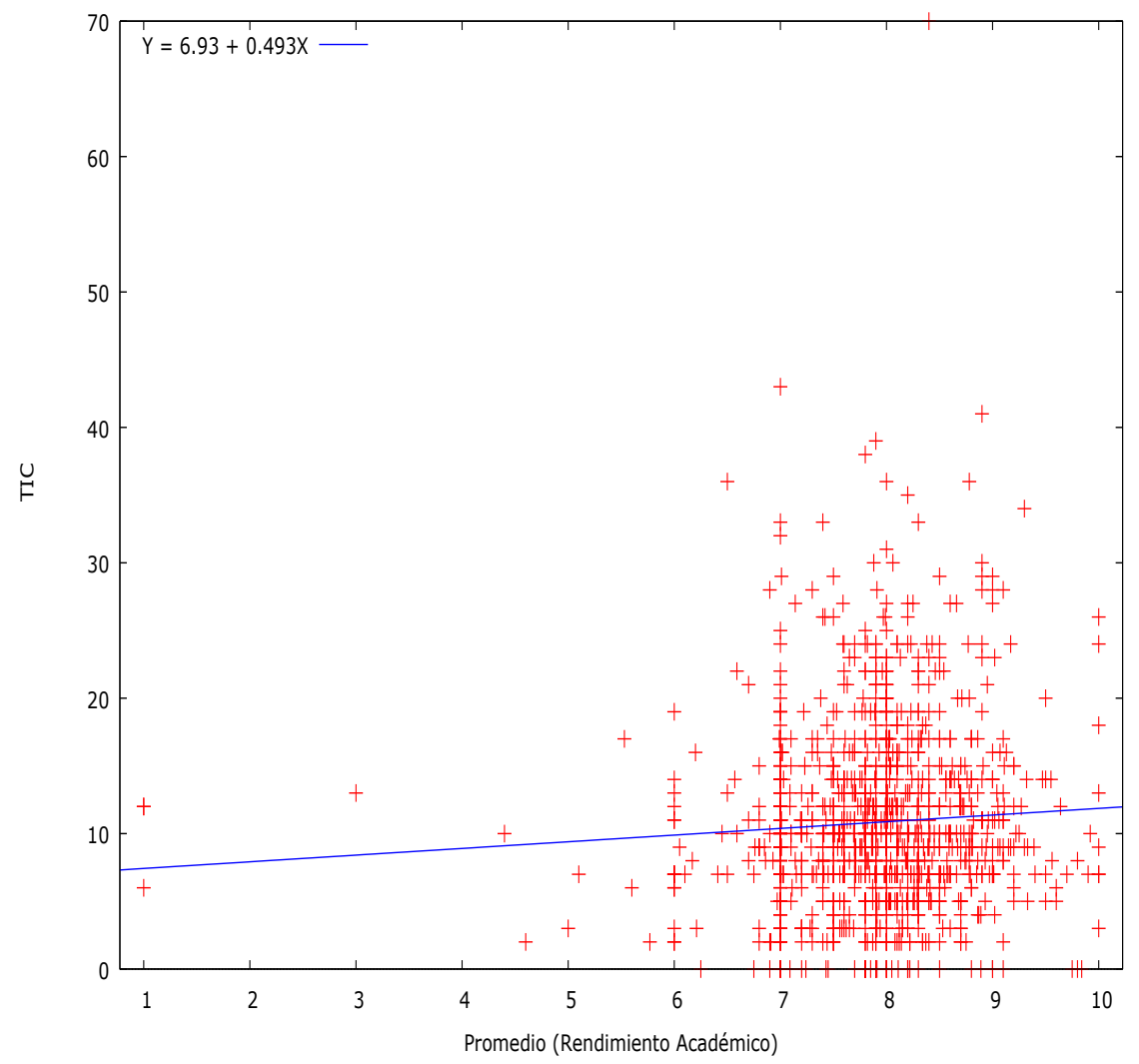

Fig. 2: Diagrama de dispersión de Promedio y TIC

En la Tabla 2 se presenta un análisis descriptivo de la base de datos empleada, con las variables que serán utilizadas en el modelo, dicha tabla incluye la media, desviación estándar, mínimo y máximo y se percibe que la variable redes sociales es la que tiene una mayor participación.

Tabla 2: Análisis descriptivo de datos

\begin{tabular}{lrrrr}
\hline & Media & Desv. Estándar & Mínimo & Máximo \\
\hline Monto de la Beca & 299,08 & 456,4 & 0 & 2190 \\
\hline Semestre & 4,18 & 2,1 & 1 & 8 \\
\hline Haiku Deck & 0,20 & 0,8 & 0 & 7 \\
\hline Sway & 0,26 & 0,9 & 0 & 7 \\
\hline Formularios de Google & 1,64 & 1,9 & 0 & 7 \\
\hline Redes Sociales & 6,38 & 3,9 & 0 & 21 \\
\hline
\end{tabular}

Se realizó una estimación del modelo de regresión Logit. Los resultados obtenidos del modelo en cuanto a coeficientes de determinación de regresión y valor $p$, se presentan en la Tabla 3.

Cabe hacer mención que los coeficientes betas estimados son estadísticamente significativos a un alfa de 0.05; por lo que se asegurar que los coeficientes de la regresión logística obtenidos en el modelo que influyen en la existencia de una beca estudiantil aportan estadísticamente a la explicación del modelo de forma adecuada por parte del estadístico valor $p$. El porcentaje de concordancia del modelo ajustado asciende a 92.3; lo que significa que el modelo ajustado es bueno, ya que explica en un $92.3 \%$ a la variable dependiente. 
En la Tabla 3, se presentan las variables semestre, Haiku Deck y Participación en Redes Sociales, y se muestran con valores positivos de $\beta$, lo cual se confirma con el exp ( $\beta$ ) mayor que 1. Lo que indica que para la variable Haiku Deck, la posibilidad de tener una beca es de aproximadamente más de 2 veces mayor [exp $(\beta)=2.462]$. Asimismo, para la Participación en Redes Sociales, la posibilidad de tener una beca es aproximadamente $11 \%$ mayor $[\exp (\beta)=1.118]$.

Tabla 3: Coeficientes de determinación del Modelo

\begin{tabular}{llllll}
\hline Variable dependiente Beca estudiantil & \multicolumn{1}{l}{ Valor $p$} & $\operatorname{Exp}(\beta)$ & \multicolumn{1}{c}{ Intervalo de Confianza (95\%) } \\
\hline & $\beta$ & & & Límite Inferior & Limite Superior \\
\hline const & -5.135 & $<0.0001$ & 0.006 & & \\
\hline MontoBeca & 0.011 & $<0.0001$ & 1.011 & 1.009 & 1.013 \\
\hline Semestre & 0.259 & 0.0073 & 1.295 & 1.072 & 1.565 \\
\hline Haiku Deck & 0.900 & $<0.0001$ & 2.462 & 1.639 & 3.699 \\
\hline Sway & -0.647 & 0.0078 & 0.523 & 0.325 & 0.843 \\
\hline Formularios de Google & -0.261 & 0.0385 & 0.770 & 0.601 & 0.986 \\
\hline Participación en Redes Sociales & 0.111 & 0.0290 & 1.118 & 1.011 & 1.235 \\
\hline Porcentaje de concordancia: 92.3\% & & & & & \\
\hline
\end{tabular}

En la Tabla 3, se puede observar que el valor negativo de $\beta$ para la variable Sway indica que los alumnos que utilizan este software disminuyen la posibilidad de obtener una beca, esto se confirma con el exp $(\beta)$ menor que 1 (0.523). Lo mismo sucede con la variable Formularios de Google, pues indica que los alumnos que utilizan este software disminuyen la posibilidad de obtener una beca, esto se confirma con el exp $(\beta)$ menor que 1 (0.770). Los valores positivos de $\beta$ y mayores que 1 del exp $(\beta)$ para las variables exógenas muestran que la posibilidad de tener una beca fue mayor para los alumnos que utilizan la herramienta informática Haiku Deck, siempre en relación con las otras herramientas de software. En cuanto al monto de la beca resulta lógico la existencia de una beca estudiantil. Con relación al Semestre, se percibe que a medida que se incremente semestre, la probabilidad de obtener la beca estudiantil es aproximadamente $3 \%$ mayor [exp $(\beta)=1.295]$.

\section{DISCUSIÓN}

Debido a que el uso de las TIC es importante como fuente de innovación y de crecimiento económico. Es importante encontrar las tecnologías que ayudan a obtener un mayor ingreso, para este caso es una beca estudiantil. En cuanto al software, derivado de esta investigación se puede observar que las herramientas para generar contenido de tipo multimedia como Haiku Deck, y la participación en las redes sociales aportan evidencia para que un alumno pueda obtener una beca estudiantil, independientemente de todos los beneficios como pueden ser un alto rendimiento académico, un aprendizaje divertido y fácil, tal y como lo señalan los autores Fong y Holland., (2011), Peeraer y Van Petegem, (2011), Alfaro et al., 2016; Martínez et al. (2015). Asimismo, las herramientas informáticas en cuanto a la participación en redes sociales también son relevantes, por lo cual es conveniente fomentarlas en el ámbito académico para que los estudiantes tengan una comunicación digital que sea oportuna con sus compañeros (Brown et al. 2016; Eid, y Al-Jabri, 2016; Čičević et al., 2016; Lee, y Bonk, 2016; Kitsantas et al., 2016), pues un alto porcentaje de alumnos en México tiene un teléfono inteligente con conectividad a internet para hacer uso de las redes sociales. Sin embargo, no se hace uso de las redes sociales, pues en ocasiones los profesores prohíben el uso del teléfono.

Por otro lado, es importante identificar las tecnologías de software con efecto positivo (Haiku Deck y Redes Sociales) y negativo (Sway y Formularios de google) en la educación, para poder formular políticas educativas que ayuden a favorecer el rendimiento académico de los alumnos. Sin descuidar el entorno social y económico al que pertenecen los alumnos (Rodriguez, 2015). Además, se debe de incluir el hardware más utilizado como es la computadora (Cabero-Almenara y Gutiérrez-Castillo, 2015) y los dispositivos móviles.

Asimismo, se coincide con los autores citados sobre la importancia del material multimedia y educación, ya que los vídeos, audios y lecturas interactivas presentan un gran impacto en el aprendizaje; asimismo la aportación de esta investigación es que el material multimedia también conduce a obtener una beca estudiantil, la cual está directamente relacionada con el rendimiento académico del alumno, pues para obtener una beca de cualquier tipo, uno de los principales requisitos es el rendimiento académico. Además, con el uso del material multimedia los alumnos tendrán clases más divertidas y podrán repasar la clase al participar en las redes sociales en cualquier momento, en cualquier lugar y en cualquier situación; lo cual generará una 
mayor productividad en los alumnos además de tener un beneficio económico al poder comunicarse de forma más eficiente para cuestiones académicas, pues por ejemplo las redes sociales permiten el intercambio de archivos así como los comentarios sobre estos archivos. Lo cual conduce directo hacia una educación de calidad.

Se sugiere también que se capacite a los docentes y se les motive con beneficios económicos y no económicos para que hagan uso de herramientas informáticas para la generación de contenido multimedia y la participación en las redes sociales, esto puede ser a través de la generación de políticas educativas mediante estímulos de productividad académica y científica. Esperando que con la capacitación en los docentes se haga más uso de las tecnologías de información y comunicación para que los docentes pueden aplicarlas en el aula y no prohibirlas.

\section{CONCLUSIONES}

De acuerdo a los resultados de este estudio, y de su discusión y análisis se pueden concluir que existen factores que aumentan la probabilidad de tener una beca estudiantil, éstos pueden ser: 1) el monto percibido de una beca; 2) el semestre; 3) el uso de la herramienta de creación del material multimedia -Haiku Deck; y 4) la participación en las redes sociales. Esto provoca también que el alumno aumente su productividad académica y obtenga un mayor ingreso al buscar una beca de estudios. Sin embargo, el uso de las herramientas informáticas para encuestas (Formularios Google) y para crear material multimedia (Sway), presentan el riesgo de no obtener una beca estudiantil, por los que se deben de buscar otros mecanismos alternativos a estas herramientas informáticas. Asimismo, en cuanto al monto percibido de una beca, los alumnos se motivan en alcanzar un mayor rendimiento académico, pues el monto de la beca aumenta con el rendimiento académico, tal es el caso de la beca Telmex.

\section{AGRADECIMIENTOS}

Agradecemos las facilidades que fueron otorgadas para la realización del presente trabajo al Instituto Politécnico Nacional-Unidad Profesional Interdisciplinaria de Ingeniería y Ciencias Sociales y Administrativas. Proyecto SIP 20161046 Las habilidades informáticas del capital humano como fuente de crecimiento económico.

\section{REFERENCIAS}

Alfaro, C., Chavarría, J., Mora, F. y Salas, O., Las posibilidades de las tecnologías multimedios en la educación matemática de Costa Rica, Uniciencia, ISSN: 2215-3470 (en línea), 20(2), 205-212, 2016. En la web: https://goo.gl/wj8nMF. Acceso: 5 de Mayo (2017)

Banco Mundial, ICT for Greater Development Impact World Bank Group Strategy for Information and Communication Technology 2012-2015, (en la web: https://goo.gl/ruuAUm, acceso: 5 de Mayo 2017), World Bank, 79 (2012)

Brown, M.G., Wohn, D.Y. y Ellison, N., Without a map: College access and the online practices of youth from low-income communities, doi: 10.1016/j.compedu.2015.10.001, Comput. Educ. (en línea), 92, 104-116 (2016)

Cabero-Almenara, J. y Gutiérrez-Castillo, J.J., De la presentación al polimedia. Una experiencia en ciencias de la educación, Rev. Educ. Cult. e Soc., ISSN: 2237-1648 (en línea), 5(4), 28-40 (2015). https://goo.gl/YzLq3z. Acceso: 5 de Mayo (2017)

Castro, P.J. y González-Palta, I.N., Percepción de Estudiantes de Psicología sobre el uso de Facebook para desarrollar pensamiento crítico, doi: 10.4067/S0718-50062016000100006, Form. Univ. (en línea), 9(1), 45-56 (2016)

Čičević, S., Samčović, A. y Nešić, M., Exploring college students' generational differences in Facebook usage, doi: 10.1016/j.chb.2015.11.034, Comput. Hum. Behav., (en línea), 56, 83-92 (2016)

Durán, B. Z., Francisco, J., López, B., Martínez, J.G. y Flores, T.G., Formación en TIC y competencia digital en la docencia en instituciones públicas de educación superior, doi: 10.18381/Ap.v9n1.922, Rev. Innovación Educ. (en línea), 9(1), 80-96 (2017)

Eid, M. I. y Al-Jabri, I.M., Social networking, knowledge sharing, and student learning: The case of university students, doi: 10.1016/j.compedu.2016.04.007, Comput. Educ., (en línea), 99, 14-27 (2016) 
Fong, R.W. y Holland, T., A Study of Teachers' Beliefs and Practices of Using Information and Communication Technology (ICT) in Classrooms, In Science Education in International Context, SensePublishers, 143-158 (2011)

Gobierno de la República, Plan Nacional de Desarrollo 2013-2018, Gobierno de la Republica, México (2013)

Hamid, S., Bukhari, S., Ravana, S.D. y ljab, M.T., Role of social media in information-seeking behaviour of international students: A systematic literature review, doi: 10.1108/AJIM-03-2016-0031, Aslib J. Inform. Manag. (en línea), 68(5), 643-666 (2016)

INSEAD, WIPO. The Global Innovation Index 2012. Stronger Innovation Linkages for Global Growth, (en línea: https://goo.gl/MP7qu7, acceso: 5 de Mayo 2017) INSEAD WIPO (2012)

Kitsantas, T., Chirinos, D.S., Hiller, S.E. y Kitsantas, A., An Examination of Greek College Students' Perceptions of Positive and Negative Effects of Social Networking Use, in Social Networking and Education, Springer International Publishing, pp. 129-143, Suiza (2016)

Lee, J. y Bonk, C.J., Social network analysis of peer relationships and online interactions in a blended class using blogs, doi: 10.1016/j.iheduc.2015.09.001, Internet High Educ., (en línea), 28, 35-44 (2016)

Mahapatra, S., An Empirical Study on Youth's Preference for Social Networking Sites, doi: 10.5539/ijbm.v11n4p274, Int. J. Bus Manag. (en línea), 11(4), 274 (2016)

Peeraer, J. y Van Petegem, P., ICT in teacher education in an emerging developing country: Vietnam's baseline situation at the start of 'The Year of ICT', doi: 10.1016/j.compedu.2010.11.015, Comput. Educ. (en línea), 56(4), 974-982 (2011)

Rodríguez, A.H., Usabilidad de un software educativo como medio instruccional para el proceso de enseñanza-aprendizaje de una asignatura, Revista Iberoamericana para la Investigación y el Desarrollo Educativo, ISSN: 2007-2619, (en línea), (11), 2015. https://goo.gl/cagst3. Acceso: 5 de Mayo (2017)

Song, H.S., Kalet, A.L. y Plass, J.L., Interplay of prior knowledge, self-regulation and motivation in complex multimedia learning environments, doi: 10.1111/jcal.12117, J. Comput. Assist Learn., 32(1), 31-50 (2016)

Tapasco, O.A. y Giraldo, J.A., Estudio Comparativo sobre Percepción y uso de las TIC entre Profesores de Universidades Públicas y Privadas, doi: 10.4067/S0718-50062017000200002, Form. Univ., 10(2), 3-12 (2017)

Venkatesh, V., Rabah, J., Fusaro, M., Couture, A., Varela, W. y Alexander, K., Factors impacting university instructors'and students'perceptions of course effectiveness and technology integration in the age of web 2.0., McGill Journal of Education/Revue des sciences de l'éducation de McGill, ISSN: 1916-0666 (en linea), 51 (1) 533-562, 2016. https://goo.gl/oWP9Ch. Acceso: 5 de Mayo (2017)

Viñas, M., Competencias digitales y herramientas esenciales para transformar las clases y avanzar profesionalmente, Ebook Academic TotemGuard, (en línea: https://goo.gl/zrP9AY, acceso 5 de mayo 2017) (2016)

Vorderer, P., Krömer, N. y Schneider, F.M., Permanently online-Permanently connected: Explorations into university students' use of social media and mobile smart devices, doi: 10.1016/j.chb.2016.05.085, Comput. Human Behav. (en línea), 63, 694-703 (2016) 
